\title{
HIV in the Kingdom of Saudi Arabia: Can We Change the Way We Deal with Co-Infections
}

This article was published in the following Dove Press journal:

Infection and Drug Resistance

\author{
Maha Al-Mozaini' \\ Tahani Alrahbeni \\ Qais Dirar ${ }^{3}$ \\ Jawaher Alotibi ${ }^{3}$
}

Abdulrahman Alrajhi $\mathbb{D}^{3}$

'Immunocompromised Host Research Section, Department of Infection and Immunity, King Faisal Specialist Hospital and Research Centre, Riyadh, Saudi Arabia; ${ }^{2}$ College of Nursing, Riyadh Elm University, Riyadh, Saudi Arabia;

${ }^{3}$ Department of Medicine, King Faisal Specialist Hospital and Research Center, Riyadh, Saudi Arabia
Correspondence: Maha Al-Mozaini

Tel +966 II 4427866

Fax +966 II 4424519

Email mmozaini@kfshrc.edu.sa

\begin{abstract}
The first incidence of acquired immunodeficiency syndrome (AIDS) from the Kingdom of Saudi Arabia (KSA) was reported back in 1984, and by the end of 2013, around 1509 patients were diagnosed with HIV infection. Recently in 2018, the Saudi ministry of health released that the incidence of HIV in Saudi Arabia is 3 cases of HIV for every 10,000 of the population. Having said that, the surveillance of HIV will face a range of challenges in KSA despite proper medical care, counseling, family planning, diagnostic, evaluation, and the use of effective anti-retroviral therapy. Patients who underwent anti-retroviral therapy showed significant reduction in morbidity as well as mortality. On the other hand, further targeted treatment and preventive strategies are warranted to control HIV co-infections in the KSA. In addition, progress towards meeting the WHO 90-90-90 goals for HIV not only at KSA but at the MENA region too, which is that of the population, $90 \%$ are diagnosed, $90 \%$ undergoing treatment, and $90 \%$ under viral control, is not being systematically monitored. In this review, we discuss the common co-infections with HIV infections that are reported in KSA, which when compared to international trends, it is similar for both viral hepatitis and tuberculosis. Although those co-infections exist, they are presented in different ratios and percentages when compared to the international reported data. These differences mandates defining and introducing new resilient methods of treatment and preventive measures. In this review, we offer an insight into healthcare policymakers to be compliant with UNAIDS 2020 vision program. We also discuss some of the gaps and recommendations to achieve the WHO 90-90-90 goal.
\end{abstract}

Keywords: human immunodeficiency virus, opportunistic infections, Saudi Arabia, AIDS, epidemiology, health policy

\section{Introduction}

Over the past decades, understanding the pathophysiology of human immunodeficiency virus (HIV) infection has been crucial in devising additional effective strategies to prevent infection. Having said that HIV infection is on decline worldwide due to early diagnosis and long-term effective use of anti-retroviral therapy. In addition, studies show that infections decreased from 3.3 million in 2002 to 1.7 million in 2019. However, there is an increase in HIV infection rates has been reported in the Middle East and North Africa (MENA), with an estimated 20,000 individuals acquiring the virus, with 8400 morbidity rates in 2018 .

The availability of combination antiretroviral therapy (ART) is increasing and progressing at a rapid rate. ${ }^{1}$ Even though with all the availability and reachability of these combinations, nearly 1.5 million deaths are attributed to HIV annually. ${ }^{1}$ The immunodeficiency caused by uncontrolled HIV infection leads to increasing the risk of 
co-infections, especially with pathogens that are normally controlled by the innate and adaptive cellular immune responses. Furthermore, administration of ART in the presence of a HIV co-infection does not always restore the natural pathogen-specific immune response to its normal levels. Here we review the leading infectious diseases that continue to cause a significant morbidity and mortality in HIV-infected individuals in the Kingdom of Saudi Arabia (KSA) such as tuberculosis (TB) and viral hepatitis.

In order to conduct this review, we searched Pubmed, science direct, google schooler, and a number of scientific databases. The search included all articles published from Saudi Arabia which were either a review, a research or case reports, with no time limits. The key words included, Saudi Arabia, HIV, co-infections, hepatitis, viral and TB.

\section{Co-Infections Involving HIV and Viral Hepatitis in the Kingdom of Saudi Arabia}

Viral co-infections in HIV/AIDS patients may result in a more rapid disease progression, which represents a growing public health concern worldwide. Pathogenic viruses that are most likely to co-infect individuals with HIV include hepatitis B virus (HBV) and hepatitis C virus
(HCV). Both viral hepatitis tends to occur in conjunction with HIV due to similar routes of transmission. Since HBV and $\mathrm{HCV}$ are endemic in $\mathrm{KSA}$, there are numerous reports describing their prevalence, clinical characteristics, and seroepidemiology. ${ }^{2}$ However, only few reports describe HBV or $\mathrm{HCV}$ co-infections in HIV-positive people in the KSA (Table 1). HCV infection has been frequently reported to be the most dominant co-infection in HIV-positive individuals followed by $\mathrm{HBV}^{3}$ A study, based on serological detection of $\mathrm{HCV}$ and HIV infections in expatriates from the Eastern region of the KSA, included 875 expatriates of both sexes who were undergoing mandatory pre-employment testing. Among the 875 samples which were screened for HCV-HIV-specific antibodies, four samples $(0.46 \%)$ were positive, but unfortunately since this study included expatriates only, then it is not a representative of the Saudi population, because expats come to KSA for a while to work, and when their contracts are concluded they leave back to their counties. ${ }^{4}$

A 20-year longitudinal study involving 341 HIVinfected patients at King Faisal Specialist hospital and research center (KFSHRC), a major tertiary medical and academic institution in Saudi Arabic, revealed the presence of $\mathrm{HCV}$ co-infection in $12 \%$ of the subjects, which is relatively close but higher from the global percentage which stands between $5 \%$ and $10 \%{ }^{5}$ The study also

Table I Reported Co-Infection with of HIV Transmission in KSA

\begin{tabular}{|c|c|c|c|c|c|}
\hline Virus & Year & $\begin{array}{l}\text { Study } \\
\text { Location }\end{array}$ & $\begin{array}{l}\text { Reported Positive for HIV/Positive } \\
\text { Screened }\end{array}$ & $\begin{array}{l}\text { Percentage of } \\
\text { Co-Infection } \\
\text { (\%) }\end{array}$ & Authors \\
\hline \multirow{4}{*}{$\begin{array}{l}\text { Mycobacterium } \\
\text { tuberculosis }\end{array}$} & 2002 & KFSHRC & $2 / 178$ & 1.1 & Alrajhi et $a^{26}$ \\
\hline & 2006 & KFSHRC & $2 / 22$ & 9 & Hakawi \& Alrajjhi ${ }^{42}$ \\
\hline & 2010 & KFSHRC & $16 / 217$ & 7.4 & Omair et $\mathrm{al}^{27}$ \\
\hline & 2014 & KFSHRC & $29 / 602$ & 16 & Al-Mozaini et al ${ }^{5}$ \\
\hline \multirow[t]{2}{*}{ Hepatitis B Virus } & 2014 & KFSHRC & ||$/ 34 \mid$ & 3 & Alhuraiji et al ${ }^{6}$ \\
\hline & 2016 & $\begin{array}{l}\text { Western } \\
\text { Province }\end{array}$ & $12 / 142$ & 8.5 & Al-Mughales ${ }^{14}$ \\
\hline \multirow[t]{3}{*}{ Hepatitis C Virus } & 2009 & Eastern Province & $4 / 875$ & 0.46 & AlZahrani $^{43}$ \\
\hline & 2014 & KFSHRC & $4 I / 34 \mid$ & 12 & Alhuraiji et $\mathrm{al}^{6}$ \\
\hline & 2016 & $\begin{array}{l}\text { Western } \\
\text { Province }\end{array}$ & $4 / 142$ & 2.8 & Al-Mughales ${ }^{14}$ \\
\hline MERS CoV & 2015 & Jeddah & $\mathrm{I} / \mathrm{I}$ & 100 & Shalhoub et al ${ }^{38}$ \\
\hline
\end{tabular}

Note: Co-infections associated with HIV-positive patients in Saudi Arabia.

Abbreviations: KFSH\&RC, King Faisal Specialist Hospital and Research center, Riyadh; KAU, King Abdulaziz University Hospital; MOH, Ministry of Health. 
reported that heterosexual transmission was the sole cause for HCV and HIV co-infection in $22 \%$ of the study population. HCV Genotype 1 was also shown as being the commonest $(44 \%)$ followed by genotype $4(15 \%))^{5}$ On the other hand, another study in 2014 reported the presence of HBV Hepatitis B surface antigen in 3\% patients in their cohort. ${ }^{6}$ Luckily this number falls way below the global percentage of HIV/HBV co-infection, which is reported to be at $10 \%{ }^{7}$

Thus, KSA studies report HCV as the most common coinfection with HIV, followed by HBV. Blood and blood product transfusion was the most common risk factor for $\mathrm{HCV}$ and HIV co-infection, occurring in $60 \%$ of patients. The prevalence of HCV and HBV infections are 20 and 10 times higher among HIV-infected patients than in the general population without HIV infection, respectively. Thus, it is acknowledged the presence of HIV co-infection with either or both HBV and HCV in KSA from the above few but conclusive studies. Additionally, it is important to understand these viral co-infections so we can direct treatment accordingly. Furthermore, it has been reported that ART combinations are highly effective in treating HIV/ $\mathrm{HBV}$ or HIV/HCV co-infections; these combinations include elbasvir/grazoprevir, which in a Phase III trial on patients with either HCV alone or co-infected with HIV, up to $95 \%$ virological control was reported. ${ }^{8,9}$ Treatment as a measure of prevention of transmission is well established for HIV; recent data from two major HIV cohort studies indicated it to be an effective approach to decreasing HCV transmission in HIVinfected patients. ${ }^{10,11}$ Studies and data from 3 large HIV clinics in London, UK, corroborates that an aggressive HIV treatment can reduce incident $\mathrm{HCV}$ infections, a $68 \%$ reduction in $\mathrm{HCV}$ infection was observed when HIV-positive patients are treated aggressively. ${ }^{12}$ It has also been reported that patients with HIV/ $\mathrm{HBV}$ and on any tenofovir ART combinations suffered HBV therapy failure although HIV is controlled; thus, it is highly recommended to avoid tenofovir in any ART combinations. ${ }^{13}$

The low percentage of hepatitis with HIV in KSA could be attributed to the fact that hepatitis is an endemic and is monitored, treated and controlled properly. It is also possible that this low percentage is due to missed diagnosis. On that account, a local retrospective study, which estimated the prevalence of diagnosed and undiagnosed co-infections among HIV, HBV, and HCV infected patients carried out in the western region of KSA as $8.5 \%$ and $2.8 \%$, respectively. ${ }^{14}$ This study points out that it is possible that a relatively low prevalence of coinfections and a significant proportion of cases remaining undiagnosed due to lack of systematic screening.
It is noteworthy to mention that there is an algorithm followed in the treatment of HBV and HCV. These guidelines are published by the Saudi Association for the Study of Liver Diseases and Transplantation (SASLT), which publishes and updates these algorithms (https://www.saudi saslt.com/saslt-9).

Finally, there are a number of factors which might contribute to a proper achievement of the WHO 90-90-90 goal. This goal, in a nutshell, is among the population, $90 \%$ are diagnosed, $90 \%$ are undergoing anti-retroviral therapy, and $90 \%$ of the treated group are under viral control. These factors include proper screening at national level with more controlled and sensitive assays. Furthermore, all studies contribute to the theory that aggressive therapy in specific combinations will either reduce the incidence of co-infection or achieve high virological control. Finally, immunization and immunization protocols that have been proven to be an effective method of prophylaxis.

\section{HIV-I and TB Co-Infections in the Kingdom of Saudi Arabia}

Tuberculosis (TB) is a major cause of mortality among people suffering from HIV. This population contributes substantially to the global TB burden, with a higher risk of progressing from TB infection to disease than HIV-negative people. ${ }^{15,16}$ In 2015, WHO launched the "End TB" strategy, setting ambitious targets of a $90 \%$ reduction in TB incidence and $95 \%$ reduction in mortality by 2035. In 2018, the UN High-Level Meeting (UNHLM) on Tuberculosis incited the global efforts to prevent TB by setting renewed targets to provide TB preventive therapy (TPT) to 30 million people by 2020 including six million PLHIV. $^{15,17}$

Tuberculosis is a chronic infectious disease in which $\mathrm{CD}^{+}$and $\mathrm{CD}^{+}$lymphocytes provide important defense in controlling infection. Thus, CD4 lymphopenia is a welldefined risk factor for the development of active TB coinfection in HIV-infected patients. A recent study reported that lung-specific chronic immune activation may be important for HIV mediated reactivation of latent $\mathrm{TB}$ infection. Since CD4R1 administration resulted in greater depletion of memory CD4 $+\mathrm{T}$ cells and preservation of effector CD4+ T cells, it is possible that this homeostasis drives the lack of reactivation in these NHPs. Importantly, it appears that HIV pathogenicity drives mechanisms such as chronic immune activation that disturb the normal homeostasis of effector CD4+ population. ${ }^{18}$ 
Evidence shows that TB preventive therapy (TPT) reduces TB incidence and mortality in HIV-positive patients up to $37 \%$ independent of ART regimens. ${ }^{19-21}$ Despite these facts, TPT introduction and increase within HIV programs, it has been unfortunately insufficient to effectively decrease the incidence of TB in HIV-positive patients. ${ }^{19,20,22-24}$ Regrettably, with all the programs it is estimated that annually there are 920,000 new TB infections among HIVpositive patients and 300,000 deaths (a third of HIV-related deaths globally in 2017), which indicates that the UN target to reduce TB deaths in HIV-positive patients by $75 \%$ by 2020 will remain elusive and unmet. ${ }^{15,25}$

In the following paragraphs, we will discuss the prevalence of HIV-TB co-infections in Saudi Arabia. A retrospective study involving 437 patients diagnosed with TB between 1995 and 2000 at KFSHRC, Riyadh, 2 patients $(1.1 \%)$ were found to have HIV-TB co-infection. Males were screened more often than females $(45 \%$ and $36 \%$, respectively). ${ }^{26}$ The same cohort study was repeated 10 years later on the same pool of patients, TB was observed in 29 patients from the total of 372 patients, which is a significant rise. ${ }^{5}$ Another observational study conducted at the same institute to evaluate the incidence of TB in people with $\mathrm{HIV}^{27}$ showed that out of 219 patients, TB was diagnosed in 16 AIDS patients (7.4\%). The incidence rate of pulmonary TB was at $762 / 100,000$ per year; for extrapulmonary TB it was at 592/100,000 per year, and despite early diagnosis and treatment for TB, 7 patients (44\%) died. $^{27}$ Therefore, among people living with HIV in KSA, TB incidence is 30 times higher than the general population. On the other hand, when comparing it to global data, it gives an alarming picture. Globally, the incidence of TB is 20 times higher only if the patient is living in area of high TB incidence such as south Africa or India, and it is considerably lower (8.9\%) in the USA. The results of the only three studies made in KSA put HIV-TB representation alarmingly higher in list of countries where TB is associated with $\mathrm{HIV}^{28}$

Instead, as observed from the above studies, all TB cases were treated as they were diagnosed, prophylaxis plays an axial role in decreasing the incidence of TB infection and development. Prevention of tuberculosis in HIV-infected patients relies on personal case findings, isoniazid prevention protocol, initiation of ART, and potentially immunization against M. tuberculosis infection. ${ }^{29}$ Preventive isoniazid therapy has been shown to be effective in HIV-positive pregnant women in order to reduce the risk of TB during pregnancy and postpartum. ${ }^{30}$ Research on TB vaccines for HIV-infected patients has focused on a prime-boost strategy using polyantigenic whole-cell inactivated vaccines. The DarDar study which was done at Dar Alsalam, Tanzania in 2010. It was a phase III trial, randomized more than $2000 \mathrm{HIV}$-infected patients with $\mathrm{CD} 4{ }^{+}$T-cell counts of at least 200 cells $/ \mu \mathrm{L}$ and a BCG scar, with five intradermal doses of a live inactivated M. vaccae vaccine compared to placebo, it was proved to be effective with a statistically significant protection against definite tuberculosis. ${ }^{31}$

In conclusion, $\mathrm{TB}$ is highly associated with HIV patients in KSA. The high percentage of TB development and death could be attributed to the ad hoc strategy of treatment and controlling whatever symptoms or infections appear. Thus, more protection and prophylaxis either therapeutic or immunological measures are highly warranted to achieve the goal for the 3rd 90 in the 90-90-90 WHO goal. In general, the appropriate way to tackle this co-infection is via the three steps, first, proper and early diagnosis, followed, regular TB therapy and management and finally, prevention protocols using the assigned TBT and safe vaccine development and application. $^{15}$

\section{Unique Cases of Co-Infections with HIV in the Kingdom of Saudi Arabia}

In addition to viral hepatitis and TB co-infections, two interesting and unique cases of HIV co-infections have also been reported in the KSA.

Nocardia wallacei was isolated from an HIV-positive patient with pulmonary infection in Southern Saudi Arabia, ${ }^{32}$ which is a rare bacterial co-infection. Nocardiosis is often missed or misdiagnosed because its identification is based on strenuous phenotypic tests. Similarly, it was reported sparsely in a number of cases globally. Until now globally there are only 5 cases reported of Nocardia wallacei co-infection in a HIVpositive patient. Interestingly, in every case, it was nearly missed. ${ }^{33-37}$ Due to its rarity and complexity of its detection, there is no clear recommendation nor protocol for its screening and therapy in HIV-positive patients.

The second unique case reported was the Middle-East Respiratory Syndrome Coronavirus (MERS-CoV) infection, which causes severe pneumonia with significant morbidity and mortality, especially in patients with multiple comorbid conditions. There was only one case of MERS-CoV co-infection documented in an HIV-infected patient. However, this patient was reported to have a successful prognosis and clinical outcome. ${ }^{38}$ Interestingly, no other cases were ever reported 
globally, that is probably due to the limitation of MERS-CoV in other countries, and it being an emerging epidemic in the Arabian Peninsula, which needs setting monitoring and therapy guidelines urgently.

\section{Conclusion}

HIV co-infections are well established within KSA, although some of the numbers fall below the global reported ratios, but all together it is still there. The contradicting numbers could be attributed to a number of factors such as the low number of studies, low screening programs or some lack of collaborations between health institutes and/or practitioners. The progress towards meeting 90-9090 goals for HIV in the MENA is not being systematically monitored. The first step in achieving the first 90 is providing proper screening programs, which are diligent and extremely sensitive. The lack of proper screening of the probable co-infections makes them harder to tackle when they are fully erupted and attacking the immunity, this leaves the practitioner with less options of therapy. Furthermore, immunization is getting more popular and proving its importance in preventing and protecting HIV patients and specifically pregnant women.
Additionally, specific ART combinations have been proven to either protect, control viral replication or contribute to therapy failure and increasing the viral load. The combination of elbasvir/grazoprevir has been proven to be highly effective and aggressive in viral control in HIV with $\mathrm{HCV}$, while virological control failure of $\mathrm{HBV}$ when tenofovir ART combinations are used. As for TB, isoniazid prevention protocol has also proved effective as a prophylaxis for both co-infected and pregnant women. Thus, more studies should be done for this issue of prophylaxis, avoiding ART failure and proper therapy protocols, rather than reporting only.

On the other hand, a number of social studies reported that the presence of a proper social/personal support system for the patients increases their education level, awareness and compliance. All this in turn participates in better prognosis of the HIV patient and it was found to decrease the incidences of picking up co-infections. ${ }^{39-41}$

Nationwide collaborations are highly recommended to provide more updated and accurate data of screening, treatment and management of both HIV and HIV coinfections. The development of a stringent national data base where all HIV patient's information, chronological

\section{HIV CO-INFECTIONS RESEARCH TRENDS IN SAUDI ARABIA}

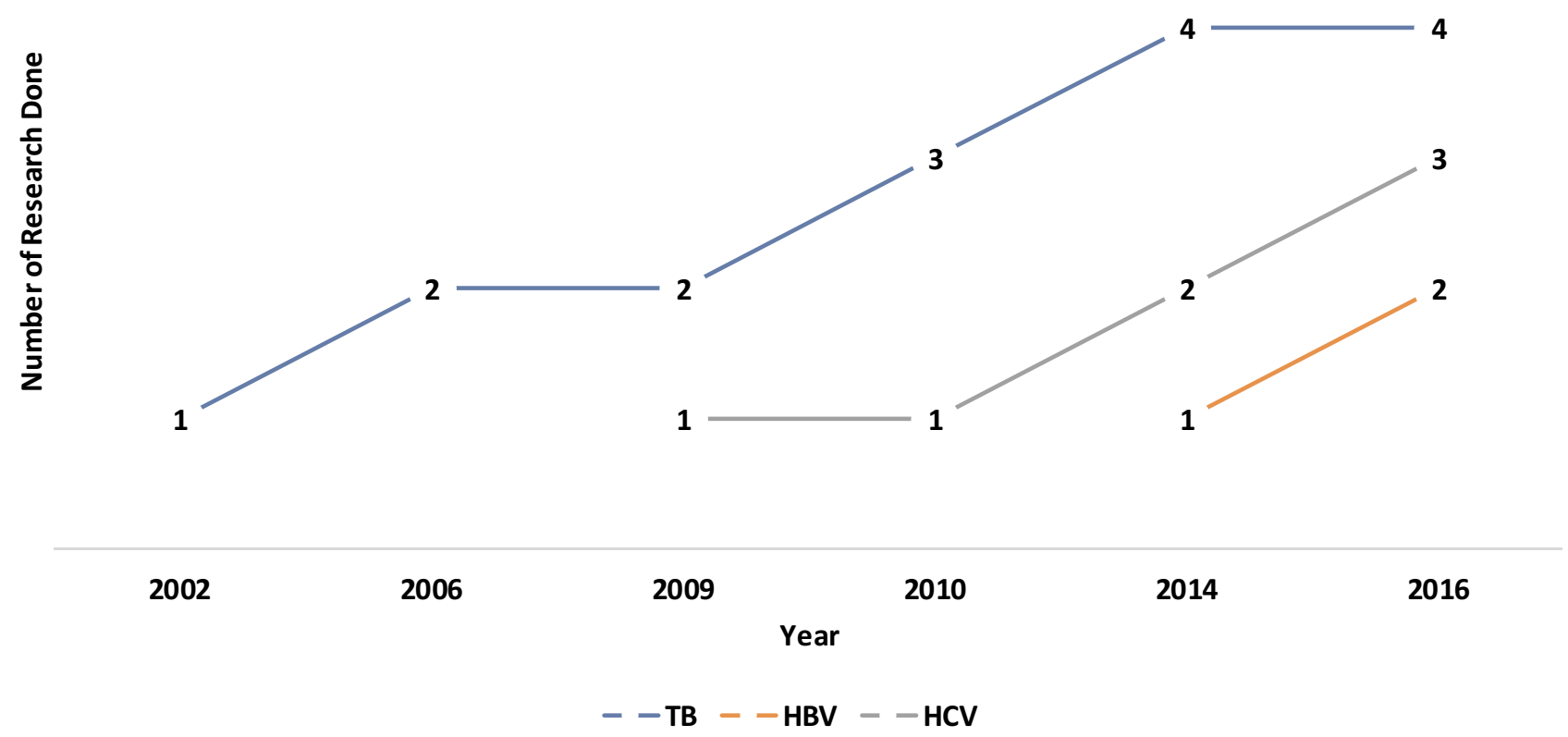

Figure I HIV co-infection research numbers and trends in Saudi Arabia. Research trends in Saudi Arabia in relation to the HIV co-infections. The blue is the HIV-TB coinfections research which started quite early at 2002 up to 2009, where it picked up pace and number. In 2009, the first HIV-HCV co-infection research was done and it followed at slow pace. The late comer into the co-infection research in Saudi Arabia was HIV-HBV co-infection, where the first research was published in 2014. 
data, types of co-infections, its management and complications. Research trends and number of studies directed specifically to HIV co-infections are increasing annually, as observed in Figure 1. The first studies were done to observe TB co-infections in 2002, and after that, the number of studies increased, HCV studies came in the picture by 2009 and $\mathrm{HBV}$ confection studies started later in 2014. All data collected will offer insight to healthcare policymakers to be compliant with UNAIDS 2020 vision program. This will also help establish the prevailing paradigms in the HIV community of aiming for AIDS-free generation and the achievement of the WHO 90-90-90 goals for diagnosis.

\section{Data Sharing Statement}

Available upon request.

\section{Acknowledgment}

We would like to thank King Abdulaziz City for Science and Technology, as a national initiative to combat emergent and re-emergent pathogen (ETSC-RKFSHRC-RCA-2017).

\section{Author Contributions}

All authors made a significant contribution to the work reported, whether that is in the conception, study design, execution, acquisition of data, analysis and interpretation, or in all these areas; took part in drafting, revising or critically reviewing the article; gave final approval of the version to be published; have agreed on the journal to which the article has been submitted; and agree to be accountable for all aspects of the work.

\section{Disclosure}

The authors declare no competing interests.

\section{References}

1. WHO. Consolidated Strategic Information Guidelines for HIV in the Health Sector. WHO; 2015.

2. Aljumah AA, Babatin M, Hashim A, et al. Hepatitis B care pathway in Saudi Arabia: current situation, gaps and actions. Saudi J Gastroenterol. 2019;25(2):73-80. doi:10.4103/sjg.SJG_421_18

3. Singh KP, Crane M, Audsley J, Avihingsanon A, Sasadeusz J, Lewin SR. HIV-hepatitis B virus coinfection: epidemiology, pathogenesis, and treatment. AIDS. 2017;31(15):2035-2052. doi:10.1097/ QAD.0000000000001574

4. Alzahrani AJ. Analysis of HIV subtypes and the phylogenetic tree in HIV-positive samples from Saudi Arabia. Saudi Med J. 2008;29 (10):1394-1396.

5. Al-Mozaini MA, Mansour MK, Al-Hokail AA, et al. HIV-care outcome in Saudi Arabia; a longitudinal cohort. J AIDS Clin Res. 2014;5 (11):11. doi:10.4172/2155-6113.1000370
6. Alhuraiji A, Alaraj A, Alghamdi S, Alrbiaan A, Alrajhi AA. Viral hepatitis B and C in HIV-infected patients in Saudi Arabia. Ann Saudi Med. 2014;34(3):207-210. doi:10.5144/0256-4947.2014.207

7. Kourtis AP, Bulterys M, Hu DJ, Jamieson DJ. HIV-HBV coinfection - a global challenge. $N$ Engl J Med. 2012;366(19):1749-1752. doi:10.1056/NEJMp1201796

8. Berenguer J, Gil-Martin A, Jarrin I, et al. Reinfection by hepatitis $\mathrm{C}$ virus following effective all-oral direct-acting antiviral drug therapy in HIV/hepatitis C virus coinfected individuals. AIDS. 2019;33 (4):685-689. doi:10.1097/QAD.0000000000002103

9. Zeuzem S, Ghalib R, Reddy KR, et al. Grazoprevir-elbasvir combination therapy for treatment-naive cirrhotic and noncirrhotic patients with chronic hepatitis C virus genotype 1, 4, or 6 infection: a randomized trial. Ann Intern Med. 2015;163(1):1-13. doi:10.7326/ M15-0785

10. Boerekamps A, van den Berk GE, Lauw FN, et al. Declining hepatitis $\mathrm{C}$ virus (HCV) incidence in dutch human immunodeficiency virus-positive men who have sex with men after unrestricted access to HCV therapy. Clin Infect Dis. 2018;66(9):1360-1365. doi:10.1093/cid/cix1007

11. Braun DL, Hampel B, Kouyos R, et al. High cure rates with grazoprevir-elbasvir with or without ribavirin guided by genotypic resistance testing among human immunodeficiency virus/hepatitis c virus-coinfected men who have sex with men. Clin Infect Dis. 2019;68(4):569-576. doi:10.1093/cid/ciy547

12. Lucy J. Fall in HCV incidence in HIV+ MSM in london following wider access to DAA therapy. CROI; 2019; Washington. 35.

13. Helen L, Zhang MM. Hepatitis B virologic failure of tenofovir-based therapies in patients with HIV/HBV. CROI; 2018; Washington: CROI.

14. Al-Mughales JA. Co-infection assessment in HBV, HCV, and HIV patients in western Saudi Arabia. $J$ Med Virol. 2016;88 (9):1545-1551. doi:10.1002/jmv.24503

15. González Fernández L, Casas EC, Singh S, et al. New opportunities in tuberculosis prevention: implications for people living with HIV. J Int AIDS Soc. 2020;23(1):e25438-e. doi:10.1002/jia2.25438

16. Pawlowski A, Jansson M, Skold M, Rottenberg ME, Källenius G. Tuberculosis and HIV co-infection. PLoS Pathog. 2012;8(2): e1002464. doi:10.1371/journal.ppat.1002464

17. Peters JS, Andrews JR, Hatherill M, et al. Advances in the understanding of Mycobacterium tuberculosis transmission in HIV-endemic settings. Lancet Infect Dis. 2019;19(3):e65-e76. doi:10.1016/S1473-3099(18)30477-8

18. Bucşan AN, Chatterjee A, Singh DK, et al. Mechanisms of reactivation of latent tuberculosis infection due to SIV co-infection. J Clin Invest. 2019;129(12):5254-5260. doi:10.1172/JCI125810

19. Johnson KT, Churchyard GJ, Sohn H, Dowdy DW. Costeffectiveness of preventive therapy for tuberculosis with isoniazid and rifapentine versus isoniazid alone in high-burden settings. Clin Infect Dis. 2018;67(7):1072-1078. doi:10.1093/cid/ciy230

20. Danel C, Moh R, Gabillard D, et al. A trial of early antiretrovirals and isoniazid preventive therapy in Africa. $N$ Engl J Med. 2015;373 (9):808-822.

21. Badje A, Moh R, Gabillard D, et al. Effect of isoniazid preventive therapy on risk of death in West African, HIV-infected adults with high CD4 cell counts: long-term follow-up of the temprano ANRS 12136 trial. Lancet Global Health. 2017;5(11):e1080-e9. doi:10.1016/S2214-109X(17)30372-8

22. Akolo C, Adetifa I, Shepperd S, Volmink J, Treatment of latent tuberculosis infection in HIV infected persons. Cochrane Database Syst Rev. 2010;(1):Cd000171. doi:10.1002/14651858.CD000171.pub3

23. Briggs MA, Emerson C, Modi S, Taylor NK, Date A. Use of isoniazid preventive therapy for tuberculosis prophylaxis among people living with HIV/AIDS: a review of the literature. $J$ Acquir Immune Defic Syndr. 2015;68(Suppl 3):S297-305. doi:10.1097/ QAI.0000000000000497 
24. Golub JE, Cohn S, Saraceni V, et al. Long-term protection from isoniazid preventive therapy for tuberculosis in HIV-infected patients in a medium-burden tuberculosis setting: the TB/HIV in Rio (THRio) study. Clin Infect Dis. 2015;60(4):639-645. doi:10.1093/cid/ciu849

25. WHO. Global Tuberculosis Report 2018. WHO; 2018.

26. Alrajhi AA, Nematallah A, Abdulwahab S, Bukhary Z. Human immunodeficiency virus and tuberculosis co-infection in Saudi Arabia. East Mediterr Health J. 2002;8(6):749-753.

27. Omair MA, Al-Ghamdi AA, Alrajhi AA. Incidence of tuberculosis in people living with the human immunodeficiency virus in Saudi Arabia. Int J Tuberc Lung Dis. 2010;14(5):600-603.

28. CDC. CfDCaP. Trends in tuberculosis-United States, 2010. MMWR Morb Mortal Wkly Rep. 2011;60(11):333-337.

29. von Reyn CF, Bakari M, Arbeit RD, Lahey T, Ramadhani A, Egwaga S. New vaccines for the prevention of tuberculosis in human immunodeficiency virus infection. Int $J$ Tuberc Lung Dis. 2012;16(6):718-723. doi:10.5588/ijtld.11.0444

30. Salazar-Austin N, Hoffmann J, Cohn S, et al. Poor obstetric and infant outcomes in human immunodeficiency virus-infected pregnant women with tuberculosis in South Africa: the Tshepiso study. Clin Infect Dis. 2018;66(6):921-929. doi:10.1093/cid/cix851

31. von Reyn CF, Mtei L, Arbeit RD, et al. Prevention of tuberculosis in Bacille Calmette-Guerin-primed, HIV-infected adults boosted with an inactivated whole-cell mycobacterial vaccine. AIDS. 2010;24 (5):675-685. doi:10.1097/QAD.0b013e3283350f1b

32. Hamid ME, Al Azraqi TA, Joseph MR, Al-Hakami AM. Isolation of a rare Nocardia wallacei from an HIV-positive patient with pulmonary infection in southern Saudi Arabia. Saudi Med J. 2013;34(6):644-647.

33. Banerjee B, Gupta R, Varma M, Mukhopadhyay C, Shaw T. Disseminated Nocardia asiatica infection in an immunocompromised individual: a rare entity needs careful vigilance. J Infect Public Health. 2019;12(2):167-170. doi:10.1016/j.jiph.2018.12.008

34. Sakyi SA, Danquah KO, Ephraim RD, et al. Evaluating the contribution of nocardia spp. and mycobacterium tuberculosis to pulmonary infections among HIV and non-HIV patients at the Komfo Anokye Teaching Hospital, Ghana. Can J Infect Dis Med Microbiol. 2018;2018:2910198.
35. Singh AK, Shukla A, Bajwa R, Agrawal R, Srivastwa N. Pulmonary nocardiosis: unusual presentation in intensive care unit. Indian J Crit Care Med. 2018;22(2):125-127. doi:10.4103/ijccm.IJCCM_472_17

36. Burke VE, Lopez FA. Approach to skin and soft tissue infections in non-HIV immunocompromised hosts. Curr Opin Infect Dis. 2017;30 (4):354-363. doi:10.1097/QCO.0000000000000378

37. Rajagopal S, Oliver NT, Palau L, Plamenac J, Mejia R. Invited commentary on treatment of Nocardia wallacei in an HIV patient with renal failure. Curr Trop Med Rep. 2016;3(2):67-70. doi:10.1007/s40475-016-0073-3

38. Shalhoub S, AlZahrani A, Simhairi R, Mushtaq A. Successful recovery of MERS CoV pneumonia in a patient with acquired immunodeficiency syndrome: a case report. J Clin Virol. 2015;62:69-71. doi:10.1016/j.jcv.2014.11.030

39. Lisa R, Metsch DJF, Masson CL, David C. Care facilitation for HIV/ $\mathrm{HCV}$ coinfected increases movement on the $\mathrm{HCV}$ care cascade. CROI; 2018; Washington: CROI.

40. Laura Starbird H-RH, Sulkowski M, Budhathoki C, Reynolds N, Farley J. A randomized trial of $\mathrm{HIV} / \mathrm{HCV}$ nurse case management for linkage to HCV care. CROI; 2018; Washington: CROI

41. John D. A community intervention increased HCV screening and treatment in King County, WA. CROI; 2018; Washington: CROI

42. Hakawi AM, Alrajhi AA. Tuberculosis of the bone marrow: clinico-pathological study of 22 cases from Saudi Arabia. Int J Tuberc Lung Dis. 2006;10(9):1041-1044.

43. Alzahrani AJ, Obeid OE, Al-Ali A, Imamwardi B. Detection of hepatitis $\mathrm{C}$ virus and human immunodeficiency virus in expatriates in Saudi Arabia by antigen-antibody combination assays. J Infect Dev Ctries. 2009;3(3):235-238. doi:10.3855/jidc.42
Infection and Drug Resistance

\section{Publish your work in this journal}

Infection and Drug Resistance is an international, peer-reviewed openaccess journal that focuses on the optimal treatment of infection (bacterial, fungal and viral) and the development and institution of preventive strategies to minimize the development and spread of resistance. The journal is specifically concerned with the epidemiology of
Dovepress

antibiotic resistance and the mechanisms of resistance development and diffusion in both hospitals and the community. The manuscript management system is completely online and includes a very quick and fair peerreview system, which is all easy to use. Visit http://www.dovepress.com/ testimonials.php to read real quotes from published authors. 